\title{
Pregnancy Outcome in Chronic Myeloid Leukemia on Imatinib Therapy - A Report of Three Patients

\author{
Geethu Babu ${ }^{1 *}$, B. Azariah², Manisha Verma ${ }^{3}$, Shruthi Prem ${ }^{4}$
}

${ }^{1}$ Assistant Professor, Department of Radiation Oncology, Regional Cancer Centre, Trivandrum-695011, India

${ }^{2}$ Assistant Professor, Department of Radiation Oncology, VMMC \& Safdarjung Hospital, New Delhi, India

${ }^{3}$ Resident, Department of Obstetrics and Gynaecology, VMMC \& Safdarjung Hospital, New Delhi, India

${ }^{4}$ Department of Medical Oncology, Regional Cancer Centre, Trivandrum-695011, India

DOI: $10.36347 /$ simcr.2020.v08i09.005

| Received: 28.08.2020 | Accepted: 05.09.2020 | Published: 09.09.2020

*Corresponding author: Geethu Babu

Abstract

Imatinib is considered as a USFDA pregnancy category D drug and contraception is advised for patients on imatinib. Here we report the pregnancy outcome of three patients, who opted to continue pregnancy while on imatinib.

Keywords: Imatinib, chronic myeloid leukemia, pregnancy.

Copyright @ 2020: This is an open-access article distributed under the terms of the Creative Commons Attribution license which permits unrestricted use, distribution, and reproduction in any medium for non-commercial use (NonCommercial, or CC-BY-NC) provided the original author and source are credited.

\section{INTRODUCTION}

CML comprises less than $10 \%$ leukemias in pregnancy and the treatment of CML in pregnancy is a clinical challenge [1]. Imatinib is classified as USFDA Pregnancy Category D based on teratogenicity reports in mice and use of effective contraception is advised in patients on imatinib. Therefore, treatment of CML in pregnancy has traditionally consisted of leukapheresis, hydroxyurea and interferon [2-4]. Since it is common practice to exclude pregnant and lactating patients from studies involving new drugs, particularly anti-cancer drugs, clinical observations are especially important.

Here we report four women with CML who became pregnant while on imatinib. They were counselled about the possible risks of discontinuing imatinib and the potential risk of fetal malformation due to imatinib. One patient who had completed her family opted for an elective medical termination of pregnancy in the first trimester. The other three patients opted to continue the pregnancy while continuing imatinib. We describe the pregnancy outcome in these three patients and also review the literature pertaining to management of CML in pregnancy. Informed consent was taken from the above patients regarding publication of this data.

\section{Patient 1}

Our first patient was a 19 year old girl who was diagnosed to have chronic phase CML in 2003 and was started on imatinib $400 \mathrm{mg} / \mathrm{day}$. After one year she had attained only a minor cytogenetic response. She progressed to lymphoid blast crisis in 2004 for which she received ALL type chemotherapy as per MCP841 Protocol and the imatinib dose was increased to $800 \mathrm{mg} /$ day. She was counselled regarding allogeneic BMT, but did not opt for the same due to financial constraints. She completed 2 yrs of maintenance chemotherapy with 6 mercaptopurve and Methotrexate in 2007. After completion of maintenance she continued $600 \mathrm{mg}$ of Imatinib per day and in 2011 was documented to have a major molecular response. She conceived in 2012 while on imatinib and received detailed counselling regarding teratogenic effects of imatinib and the danger of stopping imatinib during pregnancy (in view of past history of blast crisis). She opted to continue pregnancy while on imatinib with regular monitoring for fetal anomalies and delivered a normal healthy baby in 2012. She was advised not to breast feed the child and continues to maintain a major molecular response.

\section{Patient 2}

Our second patient was a 19 year old girl diagnosed to have chronic phase CML in 2005 for which she was started on imatinib $400 \mathrm{mg}$ / day. She had a documented major molecular response in 2012. She conceived in 2012 while on imatinib and after counselling decided to continue imatinib throughout her pregnancy. She delivered a normal healthy baby in 2013 but discontinued imatinib in order to breast feed on the advice of her gynaecologist. Three months later she presented to us with loss of hematological response and imatinib was restarted. One month after restarting 
imatinib she regained a complete hematological response.

\section{Patient 3}

Our third patient was a 23 year old lady diagnosed to have chronic phase CML in 2005 for which she was started on imatinib $400 \mathrm{mg} /$ day. After 2 years of imatinib she attained a partial cytogenetic response and a complete molecular response was documented in 2010. She conceived in 2011 while on imatinib and continued the drug throughout her pregnancy. She delivered a normal healthy baby in 2011 and did not breast feed her child. She continues to maintain complete molecular response 2 years post delivery. The growth and development of all three children have been normal till date.

\section{DisCUSSION}

Imatinib has revolutionised the treatment of CML transforming a disease with a median life expectancy of 6 to 7 years into a chronic condition with many patients leading relatively normal lives. Issues relating to the ability to conceive children therefore arise. There are two considerations in the management of a pregnant patient with CML-the mother, who needs optimal anti-cancer therapy; and the fetus, who could be affected by the disease and/or the antineoplastic drugs. Alternative drugs for CML in pregnancy include interferon and hydroxyurea. Though animal studies have shown these drugs to be nonteratogenic, interferon has been associated with abortifacient effects in animals at high doses. A more pressing concern in patients where imatinib has been stopped during pregnancy is the loss of clinical and molecular responses in patients shifted to alternative treatment.

\section{Risk to the Fetus}

Tyrosine kinases are one of the most critical groups of signaling molecules for the cellular regulation of proliferation, differentiation, and survival. Imatinib is active against the tyrosine kinases BCR-ABL, c-ABL, ARG, PDGFR, and c-KIT. Embryonic development is under complex control, and both c-KIT and PDGFR may play a role in placental development and angiogenesis. Mice homozygous for null mutations in PDGFR show combinations of birth defects, including skeletal, cardiac, neural tube, renal and urogenital anomalies [5]. Preclinical studies of organogenesis in pregnant rats showed that imatinib is teratogenic at doses equivalent to that used in humans, causing defects such a sexencephaly, encephalocele and skull bone [6]. Impaired spermatogenesis was also observed in animals [7].

However it is doubtful if animal data can be extrapolated to humans; in a series by Ault et al., male patients on imatinib were partners in eighteen pregnancies [8]. There is also no evidence that imatinib is mutagenic or clastogenic. The relation of timing of imatinib exposure in different trimesters of pregnancy to teratogenic effect is also not clear. The key period in embryogenesis is from week 3 to week 8 postconception. Hence, many authors advise against the use of anti-neoplasic agents during the first trimester. However most patients (including the three patients in our series) present with a diagnosis of pregnancy 6-8 weeks post-conception, and hence the fetus has already been exposed to imatinib during this key period. In the largest series (180 pts) by Pye et al., the timing of exposure to imatinib by trimester was available for 146 patients [9]. $103(71 \%)$ were exposed in the first trimester (including 4 exposed in both the first and second trimesters). Thirty eight (26\%) patients received the drug throughout their pregnancy includng women exposed until the time of termination of pregnancy or spontaneous abortion. An additional 4 patients were exposed only after the first trimester. Russell et al reported that imatinib crosses the mature placenta inefficiently, making it unlikely to play a significant role in the development of fetal abnormalities [10].

However, the mechanism of nutrient supply to the fetus varies at different stages of gestation. Prior to 10 weeks, there are no maternal arterial connections with the intervillous space, and the embryo obtains nutrients by simple diffusion from blood in the trophoblastic lacunae. The utero-placental and fetoplacental circulations are established after the tenth week of gestation. These differences may account for varying effects according to the time of exposure to a drug. In our study as all three patients opted to continue imatinib till delivery the fetus was exposed to imatinib throughout pregnancy.

The majority of pregnacies reported in patients on imatinib have resulted in the delivery of normal children. Ault et al., reported a series of 19 pregnancies where either the male or female partner was on imatinib [8]. Three pregnancies ended in spontaneous abortions (16\%), including two $(20 \%)$ of the ten pregnancies in female patients. This rate is somewhat higher than the reported rate of spontaneous abortions in the general population of $10 \%$ to $15 \%$ [11]. Thus, it is possible that exposure to imatinib may slightly increase the risk of spontaneous abortions. Two (13\%) of the 16 babies born from these patients had minor anomalies (one child had hypospadias and another had mild rotation of the small intestine). The authors suggest that within the constraints of a small series this could represent a higher frequency than expected of congenital abnormalities.

The largest series to date reported data on 180 women who were exposed to imatinib during pregnancy [9]. Outcome data were known for 125 (69\%) pregnancies. 63 pregnancies $(50 \%)$ resulted in the birth of normal live infants. 35 women $(28 \%)$ underwent elective terminations, 3 following the identification of fetal abnormalities. 18 pregnancies $(14 \%)$ ended in spontaneous abortion. Twelve pregnancies (9.6\%) 
resulted in infants with fetal abnormalities, 10 of the 12 infants with abnormalities are known to have been exposed to imatinib during the first trimester. Three of these infants had strikinglysimilar complex malformations that are a cause for concern- one had hypoplastic lungs, exomphalos, duplex left kidney, absent right kidney, hemivertebrae and a right shoulder anomaly; the second had exomphalos, right renal agenesis and hemivertebrae and the third child had a small exomphalos and scoliosis. Similar bony defects had also been observed in animal studies [5]. This combination of very similar, complex defects is unlikely to occur by chance and makesan imatinibinduced effect probable. Therefore the authors concluded that though most pregnancies exposed to imatinib are likely to have a successful outcome, there remained a risk of serious fetal malformations in a minority of children.

The three patients in our series were advised regarding the risk of fetal deformities due to imatinib. After detailed counselling, the patients opted to continue imatinib during the pregnancy and underwent regular sonographic screening for fetal malformations. This differs from the majority of published cases where imatinib was stopped immediately after the diagnosis of pregnancy and resumed only in the post-partum period. However despite the fetuses being exposed to imatinib throughout pregnancy, none of the infants were diagnosed with congenital malformations and have normal growth and development till date.

\section{Risk to the Mother}

Pregnancy itself does not affect the prognosis of CML [12]. However, in the series by Ault et al., of the ten female patients who interrupted imatinib therapy in pregnancy, only three achieved a complete cytogenetic response at a median of 18 months of resuming treatment after delivery while none attained a major molecular response. Moreover during pregnancy, four patients (40\%) had loss of CHR. In our series, one patient who stopped imatinib on her own during lactation had a loss of hematological response after three months despite having a major molecular response documented prior to her pregnancy, however the hematological response reverted back on restarting imatinib. The other two patients had continued imatinib throughout pregnancy and were advised not to breast feed and maintained their molecular responses as in the pre-partum period. Thus, patients who want to interrupt therapy to become pregnant should be advised of the risk of losing their clinical response.

However, not all patients lose hematological/molecular response on stopping imatinib. Rousselot et al., described 12 patients in complete molecular remission for at least 2 years, who discontinued imatinib [13] of these 12, $6(50 \%)$ patients developed molecular relapse within 5 months of stopping imatinib therapy but regained the same response within a short period after re-introduction of imatinib. The remaining six continued to have undetectable BCR-ABL transcripts at a median followup of 18 months. So the ideal patient who could be monitored off imatinib during pregnancy would be one with a sustained complete molecular response. This however occurs only in a minority of patients on imatinib and regular monitoring of bcr-abl transcript levels would have to be done in such patients to detect relapse. The advice for patients who are receiving imatinib and want to become pregnant is more difficult. Interrupting therapy for a period of time before stopping contraception may be an option. However, the duration of this interruption is debatable and depends on both the half-life of imatinib and the half-life of the sperm and ova that have been exposed to imatinib. In addition, the implications of interrupting treatment on the outcome of the disease should also be considered.

\section{CONCLUSION}

We conclude that patients with CML who are on imatinib at the time of conception can have normal children despite continuing imatinib throughout pregnancy. However, since severe fetal abnormalities have been described in a few such patients in literature and since discontinuing imatinib has been associated with loss of clinical response in pregnant and nonpregnant patients, patients receiving imatinib should be advised to practice adequate contraception. In patients who inadvertently conceive while on imatinib, decisions regarding continuation of pregnancy and/or stopping imatinib during pregnancy should be individualised in terms of the phase and aggressiveness of the disease and social and humanitarian considerations.

\section{REFERENCES}

1. Roychowdhury J, Bhattacharyya M, Kundu AK, Panfalia M. Successful pregnancy outcome in a patient of chronic myeloid leukemia without therapy. Journal of obstetrics and gynaecology of India. 2011 Oct;61(5):565-566.

2. Bazarbashi MS, Smith MR, Karanes C, Zielinski I,Bishop CR. Successful management of $\mathrm{Ph}$ chromosome chronic myelogenous leukemia with leukapheresis during pregnancy. Am J Hematol. 1991; 38(3): 235-237.

3. Celiloglu M, Altunyurt S, Undar B. Hydroxyurea treatment for chronic myeloid leukemia during pregnancy. Acta Obstet Gynecol Scand. 2000; 79(9): 803-804.

4. Baer MR, Ozer H, Foon KA. Interferon-alpha therapy during pregnancy in chronic myelogenousleukaemia and hairy cell leukaemia. Br J Haematol. 1992; 81(2): 167-169.

5. Apperley J. Issues of imatinib and pregnancy outcome. J Natl Compr Canc Netw.2009; 7(10):1050-1058. 
6. Hensley ML, Ford JM. Imatinib treatment: specific issues related to safety, fertility, and pregnancy. Semin Hematol. 2003; 40(2 Suppl 2): 21- 25.

7. Parichehre Y, Kazem P, Aghdas G, Mohammad N. The Biological Effects of Imatinib on Male Fertility of Wistar Rats. International Journal of Fertility and Sterility. 2009; 3(3): 135-142.

8. Ault P, Kantarjian H, O’Brien S, Faderl S, Beran M, Rios MB, et al. Pregnancy among patients with chronic myeloid leukemia treated with imatinib. J ClinOncol. 2006; 24(7):1204-1208.

9. Pye SM, Cortes J, Ault P, Hatfield A, Kantarjian H, Pilot R, Rosti G, Apperley JF. The effects of imatinib on pregnancy outcome. Blood, The Journal of the American Society of Hematology. 2008 Jun 15;111(12):5505-8.

10. Russell MA, Carpenter MW, Akhtar MS, Lagattuta TF, Egorin MJ. Imatinibmesylate and metabolite concentrations in maternal blood, umbilical cord blood, placenta and breast milk. J Perinatol. 2007; 27(4):241-243.

11. Weinberg CR, Wilcox AJ. Reproductive epidemiology. In: Rothman KJ, Greenland S, eds. Modern epidemiology. 2nd ed. Philadelphia, PA: Lippincott-Raven, 1998:pp.585-608.

12. Zuazu J, Julia A, Sierra J, Gl. Valentin M, Coma A, Sanz MA, Batlle J, Flores A. Pregnancy outcome in hematologic malignancies. Cancer. 1991 Feb 1;67(3):703-9.

13. Rousselot P, Huguet F, Rea D, Legros L, Cayuela JM, Maarek O, Blanchet O, Marit G, Gluckman E, Reiffers J, Gardembas M. Imatinib mesylate discontinuation in patients with chronic myelogenous leukemia in complete molecular remission for more than 2 years. Blood. 2007 Jan 1;109(1):58-60. 